\title{
Bakır(II)-Nikotinamit İçeren Yeni Kompleksin Yapısal ve Spektroskopik Özellikler Açısından İncelenmesi
}

\author{
Tuğba AYCAN ${ }^{1}$
}

How to cite: Aycan, T. (2021). Bakır(II)-Nikotinamit içeren yeni kompleksin yapısal ve spektroskopik özellikler
açısından incelenmesi Sinop Universitesi Fen Bilimleri
https://doi.org/10.33484/sinopfbd.719915

Araştırma Makalesi

Sorumlu Yazar

Tuğba AYCAN

tugba.aycan84@gmail.com

Yazara ait ORCID

T.A.: 0000-0002-5313-7807

Received: 13.04 .2020

Accepted: 18.12 .2020

\begin{abstract}
$\ddot{\mathbf{O z}}$
$\mathrm{Bu}$ çalışma $\quad\left[\mathrm{Cu}(\mathrm{na})_{2}\left(\mathrm{H}_{2} \mathrm{O}\right)_{4}\right]^{2+} \cdot\left[\mathrm{Cu}\left(\mathrm{H}_{2} \mathrm{O}\right)_{6}\right]^{2+} \cdot\left[\left(\mathrm{SO}_{4}\right)_{2}\right]^{4-} \cdot\left(\mathrm{H}_{2} \mathrm{O}\right)_{2}$ (na=nikotinamit) kompleksinin supramoleküler mimarisine odaklanmıştır. Kompleksin yapısal özellikleri, XRD, spektroskopik yöntemler, termogravimetrik ve elementel analiz ile tanımlanmıştır. Kompleksin monoklinik kristal sisteminde P2 $1 / \mathrm{c}$ uzay grubunda kristallendiği görülmüştür. Kompleks içinde iki $\mathrm{Cu}$ (II) metali vardır ve her ikisi de az bozulmuş oktahedral çevreye sahiptir. $\mathrm{Cu}$ (II) iyonlarından birisi su moleküllerinin oksijen atomlarıyla koordine iken diğeri na ligandının iki azot atomu ve su moleküllerinin oksijen atomlarıyla koordinedir. Kristal yapıda, su ve kristal örgü su moleküllerinden dolayı çok fazla hidrojen bağı vardır. Kompleks hidrojen bağları ile supramoleküler yapıyı meydana getirmiştir. Kompleksin IR spektroskopik çalışmaları, esas olarak nikotinamitin karakteristik titreşimlerine odaklanarak orta IR bölgesi içerisinde gerçekleştirilmiştir.
\end{abstract}

Anahtar Kelimeler: Tek kristal XRD, IR spektroskopisi, UV-Vis. spektroskopisi, termal analiz

\section{Investigation of the Novel Complex with Copper(II)-Nicotinamide in Terms of Structural and Spectroscopic Properties}

${ }^{1}$ Sinop Üniversitesi, Fen-Edebiyat

Fakültesi, Fizik Bölümü, Sinop, Türkiye

$\mathrm{Bu}$ çalışma Creative Commons Attribution 4.0 International License ile lisanslanmıştır

\section{Abstract}

$\left[\mathrm{Cu}(\mathrm{na})_{2}\left(\mathrm{H}_{2} \mathrm{O}\right)_{4}\right]^{2+} \cdot\left[\mathrm{Cu}\left(\mathrm{H}_{2} \mathrm{O}\right)_{6}\right]^{2+} \cdot\left[\left(\mathrm{SO}_{4}\right)_{2}\right]^{4-} \cdot\left(\mathrm{H}_{2} \mathrm{O}\right)_{2}$ (na=nicotinamide), was investigated by focusing on its supramolecular architecture. Structural properties of the complex were characterized by XRD, spectroscopic methods, thermogravimetric and elemental analysis. Further ultra-violet (UV)-visible spectral analysis and Thermogravimetric analysis were performed to understand optical and thermal properties. It has been observed that complex has crystallized in the monoclinic crystal system in $\mathrm{P} 2{ }_{1} / \mathrm{c}$ space group. The complex has two $\mathrm{Cu}(\mathrm{II})$ metals; one is coordinated with the oxygen atoms of six aqua while the other is coordinated through two nitrogen atoms of the na and the oxygen atoms from four aqua. Both $\mathrm{Cu}(\mathrm{II})$ ions have slightly distorted octahedral environments. There are a lot of hydrogen bonds due to aqua and water molecules. The complex is connected by hydrogen bonds to form a supramolecular structure. The IR spectroscopic study of the complex was carried out within the middle IR region, substantially focusing on the characteristic vibrations of nicotinamide.

Keywords: Single Crystal XRD, IR spectroscopy, UV-Vis. spectroscopy, thermal analysis 


\section{Giriș}

Nikotinamit, niyasin olarak bilinen nikotinik asidin amitidir ve suda çözünen bir vitamindir. Nikotinamit ve nikotinik asit ayn vitamin fonksiyonlarına sahip olmasına rağmen, farmakolojik ve toksik etkileri farklıdır [1]. Nikotinamit ligandı canlı hücrelerin metabolizmasında önemli bir rol oynar. Literatür araştırmaları Bakır(II) iyonunun aktivitesinin biyolojik olarak aktif ligandların ilavesiyle arttığını göstermektedir [2]. Nikotinamitin metal komplekslerinden bazıları antibakteriyel ve insülin-mimetik ajanlar olarak biyolojik olarak aktiftir [3-5].

Metal sülfatların kimyası, ilginç yapısal özellikleri, potansiyel uygulamaları, ferroelastik ve ferroelektrik özellikleri nedeniyle son yıllarda dikkat çekmiştir [6]. Metal sülfatların boyutları monomerlerden 3D polimerlere kadar değişir, hidrojen bağlarının yanı sıra su molekülleri kompleksin boyutunun artmasina katkıda bulunur [7]. Ayrica, koordinasyon eğilimi olan organik moleküllerin supramoleküler özelliklere sahip kristal katıların elde edilmesinde etkin rol oynadığ 1 bilinmektedir [8].

$\mathrm{Bu}$ çalışmada nikotinamit, B3 vitamini ailesinden seçilmiştir. Daha önceki çalışmada $\mathrm{Co}(\mathrm{II})$ ve $\mathrm{Zn}$ (II) komplekslerinin benzer çalışması yapılmıştır [9]. $\left[\mathrm{Cu}(\mathrm{na})_{2}\left(\mathrm{H}_{2} \mathrm{O}\right)_{4}\right]^{2+} .\left[\mathrm{Cu}\left(\mathrm{H}_{2} \mathrm{O}\right)_{6}\right]^{2+} \cdot\left[\left(\mathrm{SO}_{4}\right)_{2}\right]^{4-} .\left(\mathrm{H}_{2} \mathrm{O}\right)_{2}$ kompleksi sentezlenmiştir. Hidrojen bağları ile bileşiğin supramoleküler yapısı araştırılmıştır. $\mathrm{Bu}$ amaçla, kompleksin yapısal özellikleri X1şını kırınımı (XRD) tekniği, elementel analiz ve FT-IR spektroskopisi ile karakterize edilmiştir. Ek olarak, optik ve termal özellikleri UV-Vis spektroskopisi ve termogravimetrik analiz ile belirlenmiştir.

\section{Materyal ve Metot}

\section{Sentez}

Nikotinamitin sulu çözeltisi ( $1 \mathrm{mmol}, 0.12 \mathrm{~g}$ ), Bakır(II) sülfat penta-hidratın (1 mmol, $0.25 \mathrm{~g}$ ) sulu çözeltisine yavaş yavaş ilave edilmiştir ve karıştırılmıştır. Mavi karışım süzülmüştür ve kristalizasyon için bırakılmıştır. Filtrelenen ve oda sıcaklığında kristalleşmeye bırakılan çözeltiden, iki hafta sonra mavi tek kristaller elde edilmiştir. MW:779.64 g/mol, Verim: \%70.8, Element Analiz; Hesaplanan: $\mathrm{C}_{12} \mathrm{H}_{36} \mathrm{~N}_{4} \mathrm{O}_{22} \mathrm{~S}_{2} \mathrm{Cu}_{2}(\%): \mathrm{C}, 19.5 ; \mathrm{H}, 4.9 ; \mathrm{N}, 7.6$; Bulunan. C, 19.1; H, 4.6; N, 7.3.

\section{Fiziksel Ölçümler}

Tüm kimyasal malzemeler ticari şirketlerden satın alınmış ve daha fazla saflaştırılmadan kullanılmıştır. FT-IR spektrumları, orta IR bölgesinde (4000-400 $\left.\mathrm{cm}^{-1}\right)$, ATR yöntemiyle bir Bruker Tensor 27 FT-IR spektrometresinde kaydedilmiştir. Spektrumlar Bruker OPUS yazılımı kullanılarak geçirgenliğe dönüştürülmüsstür. Element analizleri $(\mathrm{C}, \mathrm{H}, \mathrm{N})$ ODTÜ Merkez Laboratuvarında bir LECO CHNS-932 elementel analiz cihazı ile yapılmıştır. Aynı anda TG, DTG ve DTA eğrileri, OMU-KITAM laboratuvarında TA DMAQ800 termal analizörü kullanılarak platin patlarda $10 \mathrm{~K} /$ dak isitma hızında $20-1000{ }^{\circ} \mathrm{C}$ sıcaklık aralığında azot atmosferinde elde edilmiştir. Kompleksin UV-Vis. spektrumu, oda sıcaklığında, 190 ila $1100 \mathrm{~nm}$ arasında 
çalışan bir Unicam UV-Vis. spektrofotometresinde su çözeltisi içinde kaydedilmiştir. Kompleksin absorbsiyon spektrumu VISIONcollect yazılımı kullanılarak çizilmiştir. XRD verileri, grafit monokromatik $\mathrm{MoK}_{\alpha}$ radyasyonu ile 296 K'de bir Stoe IPDS difraktometresi kullanılarak toplanmıştır $(\lambda=$ $0.71073 \AA$ ). Kristal yap1, doğrudan yöntemlerle analiz edilmiştir ve hidrojen olmayan tüm atomlar, SHELX97 programı kullanılarak tam matris en küçük kareler yöntemleriyle anizotropik olarak arıtılmıştır [10]. Moleküler çizimler ve diğer bilgiler için WinGX [11], ORTEP-3 [12] ve MERCURY [13] yazılımları kullanılmıştır. Kompleks için kristal verileri ve arıtım parametreleri Tablo 1'de verilmiştir.

Tablo 1. Kompleksin kristal arttım verileri

\begin{tabular}{|c|c|}
\hline & \\
\hline Formül & $\mathrm{C}_{6} \mathrm{H}_{18} \mathrm{~N}_{2} \mathrm{O}_{11} \mathrm{SCu}$ \\
\hline Ağırlık & 389.82 \\
\hline Sicaklık(K) & 296 \\
\hline Radyasyon, $\lambda\left(\mathrm{MoK}_{\alpha}\right) \AA$ & 0.71069 \\
\hline Kristal sistem & Monoklinik \\
\hline Uzay grubu & $P 2_{l} / c$ \\
\hline Birim hücre boyutları & \\
\hline$a, b, c(\AA)$ & $14.207(5), 7.678(5)$, \\
\hline$\alpha, \beta, \gamma\left({ }^{\circ}\right)$ & $15.251(5)$ \\
\hline $\operatorname{Hacim}\left(\AA^{3}\right)$ & $\begin{array}{l}90,117.678(5), 90 \\
1473.1(12)\end{array}$ \\
\hline$Z$ & 4 \\
\hline Hesaplanan yoğunluk $\left(\mathrm{Mg} \cdot \mathrm{m}^{-3}\right)$ & 1.758 \\
\hline$\mu\left(\mathrm{mm}^{-1}\right)$ & 1.68 \\
\hline$F(000)$ & 804 \\
\hline Kristal boyutu (mm) & $0.69 \times 0.42 \times 0.15$ \\
\hline$\theta$ aralığ $\left(^{\circ}\right)$ & $1.5-27.9$ \\
\hline İndeks aralığg & $\begin{array}{l}-18 \leq h \leq 18 \\
-9 \leq k \leq 9 \\
-19 \leq l \leq 19\end{array}$ \\
\hline Ölçülen yansımalar & 22986 \\
\hline Bağımsız yansımalar & 3314 \\
\hline Gözlenen yansımalar $[I \geq 2 \sigma(I)]$ & 2372 \\
\hline Absorbsiyon düzeltmesi & integrasyon \\
\hline Aritım metodu & $\begin{array}{l}\text { Tam matris en küçük } \\
\text { kareler de } F^{2}\end{array}$ \\
\hline Veri/Kisitlamalar/Parametreler & $3314 / 0 / 249$ \\
\hline$S$ & 0.93 \\
\hline Son R indeksi [ $I \geq 2 \sigma(I)]$ & $\begin{array}{l}\mathrm{R}_{1}=0.042 ; \mathrm{wR}_{2}= \\
0.121\end{array}$ \\
\hline$R$ indisleri (tüm veri) & 0.085 \\
\hline$\Delta \rho_{\operatorname{maks}} ; \Delta \rho_{\min }\left(\mathrm{e} \AA^{-3}\right)$ & $0.73 ;-0.52$ \\
\hline
\end{tabular}

\section{Tartışma}

\section{Kompleksin Kristal ve Moleküler Yapısı}

Şekil l'de gösterildiği gibi, kompleks iki bakır iyonuna sahip bir monomerdir. $\mathrm{Cu} 1$ iyonu, iki nikotinamitin piridin halkasındaki azot atomu ve dört su molekülünün oksijen atomu ile koordine olurken, $\mathrm{Cu} 2$ iyonu alt1 su molekülünün oksijen atomuna koordine olmaktadır.

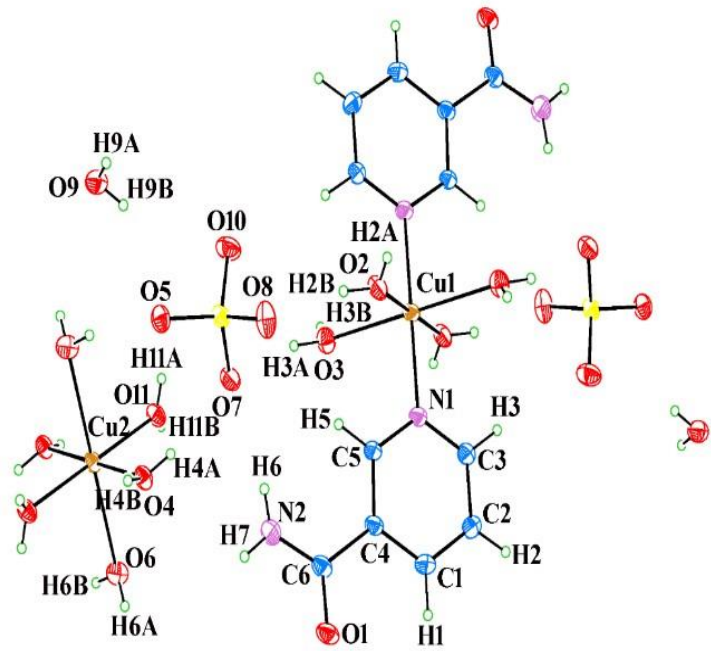

Şekil 1. Kompleksin moleküler yapısı. Yer değiştirme elipsoitleri \%20 oranında çizilmiştir.

$\mathrm{Cu}$ (II) iyonlarının koordinasyon polihedronu hafifçe bozulmuş oktahedral geometriye karş11ık gelir. $\mathrm{Cu} 1$ katyonunun ekvatoral pozisyonu, iki su molekülünün oksijen atomları ve iki na ligantının azot atomları tarafından işgal edilirken, eksensel pozisyonlar iki su molekülünün oksijen atomları tarafından işgal edilir. 
Tablo 2. Kompleksin seçilen bağ uzunlukları (Å) ve bağ açıları $\left(^{\circ}\right)$

\begin{tabular}{|c|c|c|c|c|}
\hline & Kompleks & [14] & [15] & [6] \\
\hline \multicolumn{5}{|c|}{ Bağ uzunlukları $(\AA)$} \\
\hline Cu1-N1/N1ii & $2.003(2)$ & $2.012(2)$ & $2.016(2)$ & - \\
\hline $\mathrm{Cu} 1-\mathrm{O} 3 / \mathrm{O}^{3 i \mathrm{i}}$ & $2.037(2)$ & $1.983(2)$ & $1.949(2)$ & - \\
\hline $\mathrm{Cu} 1-\mathrm{O} 2 / \mathrm{O} 2^{\mathrm{ii}}$ & $2.287(3)$ & $2.460(2)$ & 2.651(3) & - \\
\hline $\mathrm{Cu} 2-\mathrm{O} 4 / \mathrm{O} 4^{\mathrm{i}}$ & $1.963(2)$ & - & - & $1.974(2)$ \\
\hline $\mathrm{Cu} 2-\mathrm{O} 11 / \mathrm{O} 11^{\mathrm{i}}$ & $1.972(2)$ & - & - & $1.980(2)$ \\
\hline $\mathrm{Cu} 2-06 / \mathrm{O6}^{\mathrm{i}}$ & 2.404(3) & - & - & $2.395(2)$ \\
\hline S1-O10 & $1.464(2)$ & - & - & $1.464(3)$ \\
\hline S1-O5 & $1.465(2)$ & - & - & $1.470(2)$ \\
\hline S1-O7 & $1.470(2)$ & - & - & $1.475(2)$ \\
\hline S1-O8 & $1.470(2)$ & - & - & $1.478(2)$ \\
\hline \multicolumn{5}{|l|}{ Bağ açıları ( $\left.{ }^{\circ}\right)$} \\
\hline N1-Cu1-O3 & $88.74(9)$ & $89.48(8)$ & $89.10(7)$ & - \\
\hline $\mathrm{N} 1-\mathrm{Cu} 1-\mathrm{O} 2$ & $88.17(9)$ & $86.87(8)$ & $88.46(8)$ & - \\
\hline $\mathrm{O} 3-\mathrm{Cu} 1-\mathrm{O} 2$ & 94.64(11) & $94.48(8)$ & $98.97(7)$ & - \\
\hline $\mathrm{N} 1-\mathrm{Cu} 1-\mathrm{O} 3^{\mathrm{ii}}$ & $91.26(9)$ & $90.52(8)$ & - & - \\
\hline $\mathrm{N} 1-\mathrm{Cu} 1-\mathrm{O} 2^{\mathrm{ii}}$ & 91.83(9) & $93.13(8)$ & - & - \\
\hline $\mathrm{O} 3-\mathrm{Cu} 1-\mathrm{O} 2^{\mathrm{ii}}$ & $85.36(11)$ & $85.52(8)$ & - & - \\
\hline O4-Cu2-O11 & $92.14(10)$ & - & - & $86.14(8)$ \\
\hline O11-Cu2-O6 & $88.18(10)$ & - & - & $90.25(8)$ \\
\hline O4-Cu2-O6 & $92.10(11)$ & - & - & $91.21(8)$ \\
\hline O4-Cu2-O1 $1^{\mathrm{i}}$ & $87.86(10)$ & - & - & $93.86(8)$ \\
\hline O11-Cu2-O6 ${ }^{\mathrm{i}}$ & 91.82(10) & - & - & $89.75(8)$ \\
\hline $\mathrm{O} 4-\mathrm{Cu} 2-\mathrm{O}^{\mathrm{i}}$ & $87.90(11)$ & - & - & $88.79(8)$ \\
\hline
\end{tabular}

Simetri kodları: (i): $-x,-y,-z+1$; (ii): $-x-1,-y+1,-z+1$

Tablo 2'de gösterildiği gibi, Jahn-Teller bozulması olarak bilinen çok az bozulmuş oktahedral geometriden dolay1 $\mathrm{Cu} 1-\mathrm{O} 2 / \mathrm{O} 2^{\mathrm{ii}}$ (2.287 (3) Å) bağ uzunlukları diğerlerinden daha uzundur (Cu1-O3 (2.037 (2) $\AA$ ) ve Cu1N1 (2.003 (2) $\AA$ )). Kompleksin geometrik parametreleri $\left[\mathrm{Cu}\left(\mathrm{NC}-\mathrm{C}_{6} \mathrm{H}_{4} \mathrm{COO}\right)_{2}\left(\mathrm{C}_{6} \mathrm{H}_{6} \mathrm{~N}_{2} \mathrm{O}\right)_{2}\left(\mathrm{H}_{2} \mathrm{O}\right)_{2}\right.$ ] [14], $\left[\mathrm{Cu}\left(\mathrm{H}_{2} \mathrm{pm}\right)(\mathrm{na})_{2}\left(\mathrm{H}_{2} \mathrm{O}\right)_{2}\right]_{n} \cdot 4 \mathrm{nH}_{2} \mathrm{O}$ [15] ve [6] karşılaştırmalı olarak Tablo 2'de verilmektedir.

Kompleks nicotinamit ligantının amit grubu, sülfat anyonu ve su molekülleri aralarında O$\mathrm{H} \cdots \mathrm{O}$ ve $\mathrm{N}-\mathrm{H} \cdots \mathrm{O}$ hidrojen bağı ile bağlanmaktadır. Böylece $R_{6}^{6}(16), R_{2}^{1}(10)$ ve $R_{2}^{2}(8)$ halkaları meydana gelir ve bu halkaların merkezleri sirasiyla $(n, \quad 1 / 2, \quad 1 / 2 ; n=0 \quad$ veya tamsay1), $(n+1 / 4,1 / 2,1 / 2 ; n=0$ veya tamsay $)$ ve $(\mathrm{n}+1 / 5,2 / 5,3 / 5 ; \mathrm{n}=0$ veya tamsayı) konumlarında yer alır [16-17]. Bu halka zincirleri [100] doğrultusu boyunca ilerleyen 1D polimerik yapı oluşturur (Şekil 2).

$\left[\mathrm{Cu}\left(\mathrm{H}_{2} \mathrm{O}\right)_{6}\right]^{2+}$ katyonu, sülfat anyonu ve su molekülleri arasında oluşan $\mathrm{O}-\mathrm{H} \cdots \mathrm{O}$ hidrojen bağları [001] yönünde 1D zincirler oluşturur (Şekil 3b). Ek olarak, $\left[\mathrm{Cu}(\mathrm{na})_{2}\left(\mathrm{H}_{2} \mathrm{O}\right)_{4}\right]^{2+}$ katyonundaki bağlı su ve na ligantının karbonil gruplar1 arasındaki $\left(\mathrm{O} 3-\mathrm{H} 3 \mathrm{~B} \cdots \mathrm{O} 1^{\text {iv }}\right.$ iv: $x,-$ $y+1 / 2, z-1 / 2) \mathrm{f}$-bağ 1 da $1 \mathrm{D}$ zincirleri oluşturur. Her iki zincir de sülfat ve amit grubu tarafından 
oluşturulan molekül içi H-bağları (O2-H2B-O7, O3-H3A-O8) ve moleküller arası H-bağları

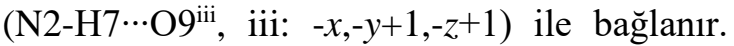

Böylece, (101) düzleminde 2D supramoleküler bir tabaka oluşur (Şekil 3a).

Tablo 3. Kompleksin hidrojen băg geometrisi $\left(\AA,{ }^{\circ}\right)$

\begin{tabular}{|c|c|c|c|c|}
\hline $\mathrm{D}-\mathrm{H} \cdots \mathrm{A}$ & D-H & $\mathrm{H} \cdots \mathrm{A}$ & $\mathrm{D} \cdots \mathrm{A}$ & $\mathrm{D}-\mathrm{H} \cdots \mathrm{A}$ \\
\hline $\mathrm{O} 4-\mathrm{H} 4 \mathrm{~A} \cdots \mathrm{O} 5^{\mathrm{iii}}$ & $0.76(4)$ & $1.98(4)$ & $2.728(3)$ & $168(4)$ \\
\hline O6-H6A $\cdots$ O10 ${ }^{\text {iv }}$ & $0.80(5)$ & $2.13(5)$ & $2.924(4)$ & $172(5)$ \\
\hline O6-H6B $\cdots{ }^{\mathrm{O}} 5^{\mathrm{i}}$ & $0.78(5)$ & $2.34(5)$ & $3.116(4)$ & $171(5)$ \\
\hline O9-H9B $\cdots \mathrm{O}^{2} 0^{v}$ & $0.89(5)$ & $1.96(5)$ & $2.842(4)$ & $170(4)$ \\
\hline $\mathrm{O} 2-\mathrm{H} 2 \mathrm{~A} \cdots \mathrm{O} 1^{\mathrm{vi}}$ & $0.67(4)$ & $2.12(4)$ & $2.780(4)$ & $169(4)$ \\
\hline $\mathrm{O} 3-\mathrm{H} 3 \mathrm{~B} \cdots \mathrm{O} 1^{\mathrm{vii}}$ & $0.69(3)$ & $2.04(3)$ & $2.731(3)$ & $175(4)$ \\
\hline $\mathrm{N} 2-\mathrm{H} 7 \cdots \mathrm{O} 9^{\mathrm{iii}}$ & $0.77(4)$ & $2.29(4)$ & $3.052(4)$ & $170(3)$ \\
\hline O11-H11A $\cdots$ O $8^{\text {iii }}$ & $0.75(4)$ & $1.89(4)$ & $2.625(3)$ & $164(4)$ \\
\hline N2-H6 $\cdots \mathrm{O} 7$ & $0.79(7)$ & $2.51(6)$ & $3.251(4)$ & $156(5)$ \\
\hline $\mathrm{O} 3-\mathrm{H} 3 \mathrm{~A} \cdots \mathrm{O} 8$ & $0.81(4)$ & $1.88(4)$ & $2.676(3)$ & $169(3)$ \\
\hline $\mathrm{O} 2-\mathrm{H} 2 \mathrm{~B} \cdots \mathrm{O} 7$ & $0.84(4)$ & $2.01(4)$ & $2.820(3)$ & $161(4)$ \\
\hline O9-H9A $\cdots$ O5 & $0.72(5)$ & $2.19(5)$ & $2.820(4)$ & $147(5)$ \\
\hline $\mathrm{O} 4-\mathrm{H} 4 \mathrm{~B} \cdots \mathrm{O} 7$ & $0.76(4)$ & $1.95(4)$ & $2.701(3)$ & $168(4)$ \\
\hline
\end{tabular}

Simetri kodlar1: (i): $-x,-y,-z+1$; (iii): $-x,-y+1,-z+1$; (iv): $x,-y+1 / 2, z-1 / 2 ;$ (v): $-x, y+1 / 2,-z+3 / 2$; (vi): $x,-y+1 / 2, z+1 / 2$; (vii): $x,-y+3 / 2, z+1 / 2$.

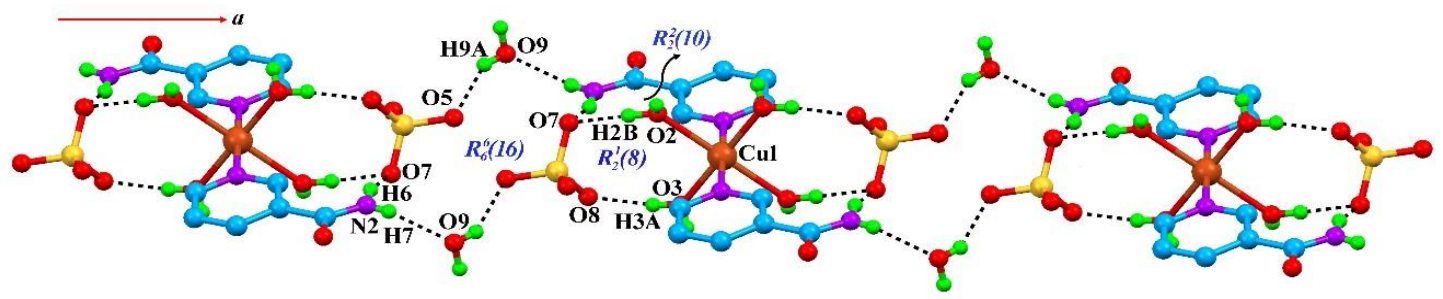

Şekil 2. Koordinasyon monomerlerinin, $R_{6}^{6}(16), R_{2}^{1}(8)$ ve $R_{2}^{2}(10)$ halkalart ile sonuçlanan $N-H \cdots O$ ve $\mathrm{O}-\mathrm{H} \cdots \mathrm{O}$ bağları ile bağlanması. İlgili olmayan $\mathrm{H}$ atomlarl ve $\mathrm{Cu}\left(\mathrm{H}_{2} \mathrm{O}\right)_{6} \mathrm{klsml}$, daha net bir görüntü için çıkarılmıştır.

Sülfat ve su molekülünün $\mathrm{O}$ atomları, $\mathrm{O}-\mathrm{H} \cdots \mathrm{O}$ H tipi hidrojen bağları ile bağlanır ve zikzak zincirleri meydana getirir (Şekil 4a). Şekil 4b 'de görüldüğü gibi, bu zincirler $\left[\mathrm{Cu}(\mathrm{na})_{2}\left(\mathrm{H}_{2} \mathrm{O}\right)_{4}\right]^{2+}$ katyonlarını ab düzleminde iki boyutlu bir ağ oluşturacak şekilde birbirine bağlar. Sonuç olarak A $\left(R_{6}^{6}(18)\right)$ halkaları $\left(\mathrm{n}_{1}\right.$, $\mathrm{n}_{2}, 1 / 2 ; \mathrm{n}=0$ veya tamsayı) konumlarında, B
$\left(R_{4}^{4}(12)\right)$ halkalar1 $\left(\mathrm{n}_{1}, \mathrm{n}_{2}+1 / 2,1 / 2 ; \mathrm{n}=0\right.$ veya tamsayı) konumlarında ve $\mathbf{C}\left(R_{2}^{2}(8)\right)$ halkaları $\left(\mathrm{n}_{1}+1 / 5, \quad \mathrm{n}_{2}+4 / 5, \quad 3 / 5 ; \quad \mathrm{n}=0 \quad\right.$ veya tamsay $)$ konumlarında yer alır. $a$-doğrultusundan bakıldığında, moleküler tabakalar Şekil 4c'de görüldüğ̈̈ gibidir ve $R_{4}^{2}(12)$ halkaları $\left(1 / 2, \mathrm{n}_{1}\right.$, $\mathrm{n}_{2}+1 / 2 ; n=0$ veya tamsayı) pozisyonlarında yer alır. Böylece, 3D supramoleküler yapı oluşur. 


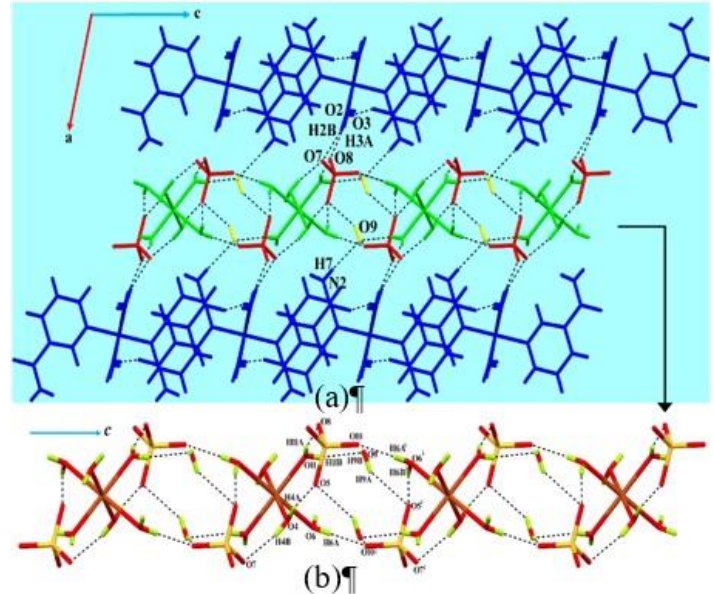

Şekil 3. (a) 1D polimerik zincirlerinin (Şekil 2), $O-H \cdots O$ hidrojen bağlart ile meydana getirdiği $2 \mathrm{D}$ tabakalar (b) $\left[\mathrm{Cu}\left(\mathrm{H}_{2} \mathrm{O}\right)_{6}\right]^{2+}$ katyonları arasinda oluşan $\mathrm{O}-\mathrm{H} \cdots \mathrm{O}$ tipi hidrojen bağlarının [001] yönündeki zincirleri.

Daha net bir görüntü için ilgili olmayan su molekülleri çıkarılmıştır. Simetri kodları Tablo 3'te verilmiştir.

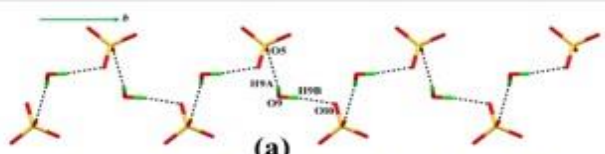

(a)
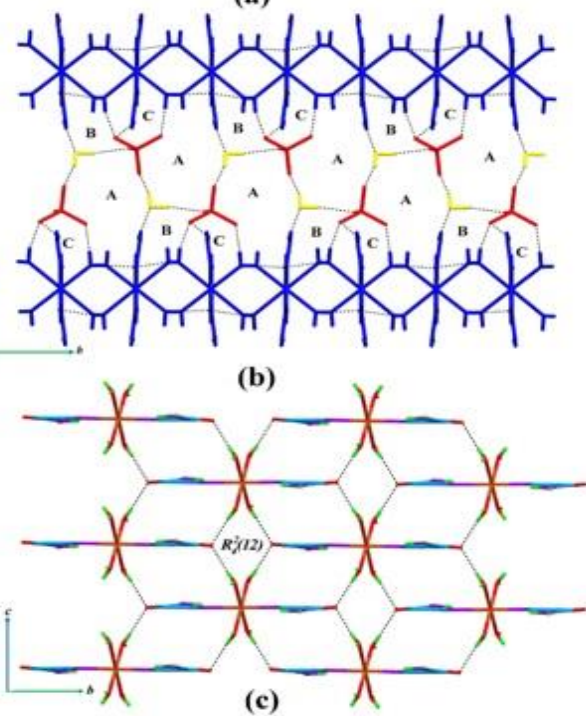

Şekil 4. (a) Sülfat ve su molekülünün $O$ atomlarının H-bağlart yoluyla bağlanmasını gösteren zig-zag zincirleri. (b) bu zincirler ile $\left[\mathrm{Cu}(\mathrm{na})_{2}\left(\mathrm{H}_{2} \mathrm{O}\right)_{4}\right]^{2+}$ katyonlartnı birbirine bağlayan, komşu $\boldsymbol{A}\left(R_{6}^{6}(18)\right), \boldsymbol{B}\left(R_{4}^{4}(12)\right)$ ve $\boldsymbol{C}\left(R_{2}^{2}(8)\right)$ halkaları. (c) $3 D$ supramoleküler yapının $x$-doğrultusundan bakıldı̆̆ında görünüşü

\section{Titreșim Spektrumu}

Kompleksin titreşim spektroskopi çalışması, esas olarak kompleks ile serbest na ligant arasındaki titreşim piklerindeki değişikliklere odaklanarak gerçekleştirilmiştir (Şekil 5). Nikotinamit molekülleri hidrojen bağları yapmak için aktif üç donör alanına sahiptir. Bu bölgeler, piridin halkasının azotu, amit grubunun primer amin ve karbonil $\mathrm{C}=\mathrm{O}^{\prime}$ dur. $\mathrm{Bir}$ amit grubunun karbonil oksijen yoluyla metal koordinasyonu, karbonil grubunun germe frekansında daha düşük bir değere kayma ile ortaya çıkar [18].

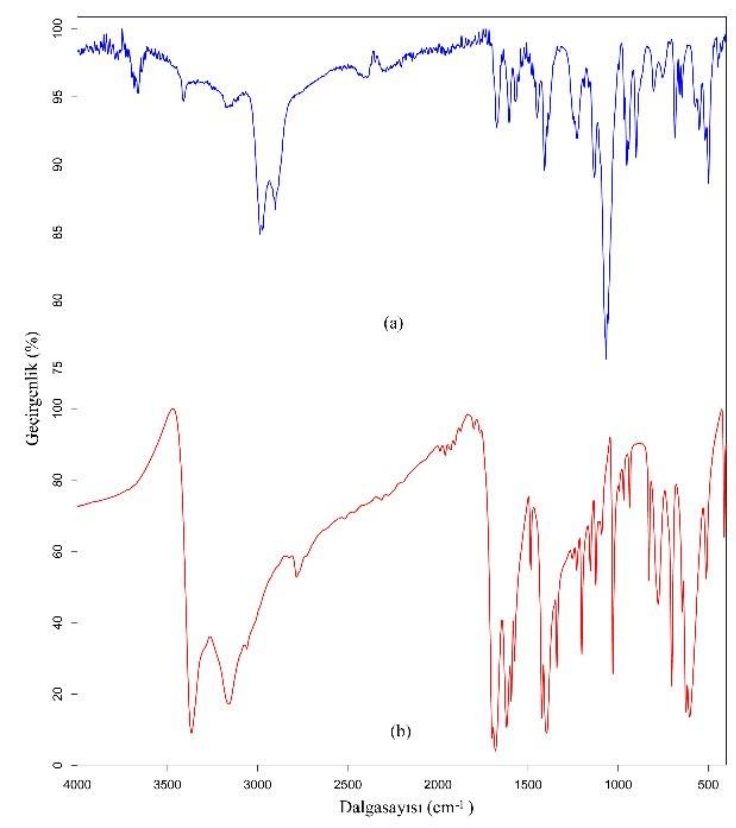

Şekil 5. (a) Kompleksin (b) Nikotinamitin FTIR spektrumlarl

\section{O-H ve $\mathrm{NH}_{2}$ Titreşimleri}

Kompleks, $\mathrm{OH}$ su grubu ve su moleküllerinden kaynaklanan $3682 \mathrm{~cm}^{-1} 3663 \mathrm{~cm}^{-1}$ ve $3514 \mathrm{~cm}^{-}$ 'de OH gerilme modlarına sahiptir. Tablo 4'te görüldüğg̈ gibi, $v\left(\mathrm{NH}_{2}\right)_{\mathrm{s}}$ ve $v\left(\mathrm{NH}_{2}\right)_{\text {as }}$ titreşim pikleri, orta kuvvettedir ve hidrojen bağı nedeniyle yüksek frekans bölgesine doğru 
kaymaktadır. Serbest $n a$ ligantında $v\left(\mathrm{NH}_{2}\right)_{\mathrm{s}}$ ve $\mathrm{v}\left(\mathrm{NH}_{2}\right)_{\text {as }}$ pikleri, $3368-3163 \mathrm{~cm}^{-1}$ aralığında güçlü pikler olarak ortaya çıkar [19]. Bu titreşim pikleri, kompleks de $3410 \mathrm{~cm}^{-1}$ ve 3170 $\mathrm{cm}^{-1}$ de gözlenir. Kompleks oluşum durumunda değerlerin değiştiği görülür. Kompleks halinde bu değerlerde bir miktar kayma görülmektedir. $\mathrm{Bu}$ durum metal-ligant koordinasyonu veya hidrojen bağından kaynaklanmaktadır.

\section{C-H, C-C ve C-N Titreşimleri}

Piridinin C-H germe titreşimi genellikle C-H germe titreşiminin karakteristik bölgesi olan 3100-3000 $\mathrm{cm}^{-1}$ aralığında görülür [20]. Buna göre, FT-IR spektrumunda $\mathrm{C}-\mathrm{H}$ simetrik gerilme titreşimleri, komplekste sırasıyla 3075 $\mathrm{cm}^{-1}$ ve $3060 \mathrm{~cm}^{-1}$ de gözlenir. Bu değerler literatür tarafından desteklenmektedir [21]. C-H düzlem içi bükme atamaları 1000-1300 $\mathrm{cm}^{-1}$ aralığında görülür [22]. Düzlem içi C-H bükülme titreşimi, kompleks de $1252 \mathrm{~cm}^{-1}$ de, serbest $n a$ da ise $1254 \mathrm{~cm}^{-1}$ de görünmektedir. Bu titreşimde gözlenen değişiklik, hidrojen bağı veya koordinasyon bağı olmadığından çok küçüktür. C-H düzlem dışı bükme atamaları 1000-500 $\mathrm{cm}^{-1}$ bölgesinde gerçekleşir [20]. Aromatik $\mathrm{CH}$ düzlem dışı bükülme bantları, kompleksin FT-IR spektrumunda $949 \mathrm{~cm}^{-1}, 802$ $\mathrm{cm}^{-1}, 752 \mathrm{~cm}^{-1}$ ve $510 \mathrm{~cm}^{-1}$ de görünür. Buna göre, na ligantında $\mathrm{CH}$ düzlem dışı bükülme titreşimleri $970 \mathrm{~cm}^{-1}, 936 \mathrm{~cm}^{-1}, 829 \mathrm{~cm}^{-1}, 778$ $\mathrm{cm}^{-1}, 517 \mathrm{~cm}^{-1}$ ve $499 \mathrm{~cm}^{-1}$ de görünür. C-C gerilme titreşim bantları 1650-1200 $\mathrm{cm}^{-1}$ aralığında meydana gelir ve $\mathrm{C}-\mathrm{N}$ germe titreşimi genellikle aromatik bileşikler için $1400-1200 \mathrm{~cm}^{-1}$ bölgesinde yer alır [20]. Piridin halkaları için $v(\mathrm{C}=\mathrm{C})+\mathrm{v}(\mathrm{C}=\mathrm{N}) \quad$ bantları komplekste $1592 \mathrm{~cm}^{-1}$ ve $1573 \mathrm{~cm}^{-1}$ de gözlenir. Ayrıca C-C ve C-N'nin diğer titreşimleri Tablo 4'te verilmiştir. Piridin halkalarının $\mathrm{C}-\mathrm{C}$ ve $\mathrm{C}-$ $\mathrm{N}$ gerilme titreşimlerinde de küçük değişiklikler görülmektedir [23].

\section{S-O Titreşimleri}

$\mathrm{SO}_{4}{ }^{2-}$ iyonlarının $\mathrm{S}-\mathrm{O}$ gerilme titreşimleri genellikle 1130-1080 $\mathrm{cm}^{-1}$ bölgesinde görülür [24]. Komplekste S-O gerilme titreşimleri 1101 $\mathrm{cm}^{-1}$ de görülür. $\mathrm{Bu}$ değer literatür ile uyumludur [25]. Literatürde $\mathrm{SO}_{4}{ }^{2-}$ iyonlarının S-O bükülmesine bağlı bantlar $680-610 \mathrm{~cm}^{-1}$ de görülmektedir [20]. Kompleksin spektrumunda ise bu bantlar $684 \mathrm{~cm}^{-1}$ de ortaya çıkmaktadır.

\section{$\mathrm{C}=\mathrm{O}$ Titreşimleri}

Serbest nicotinamit de, karbonil grubunun $v(\mathrm{C}=\mathrm{O})$ titreşimleri $1697 \mathrm{~cm}^{-1}$ ve $1679 \mathrm{~cm}^{-1} \mathrm{de}$ görülmektedir. Komplekste ise $v(\mathrm{C}=\mathrm{O})$ gerilme titreşimleri $1725 \mathrm{~cm}^{-1}$ de meydana gelmektedir. Buna göre, kompleksin spektrumları ile serbest $n a$ arasındaki bu kaymaların sebebi, hidrojen bağ1 yapmasından dolayı $\mathrm{C}=\mathrm{O}$ çift bağ karakterinin zayıflamasıdır. 
Tablo 4. Kompleksin bazı karakteristik titreşim pikleri $\left(\mathrm{cm}^{-1}\right)$ ve literatür değerleri

\begin{tabular}{|c|c|c|c|c|c|}
\hline Titreşimler & $\mathrm{Na}[18]$ & Kompleks & $\mathrm{Cu}-\mathrm{Na}$ [19] & $\mathrm{Cu}-\mathrm{Sac}-\mathrm{Na}[5]$ & $\mathrm{Cu}-\mathrm{SO}_{4}[24]$ \\
\hline$v(\mathrm{OH})$ & - & $3682,3663,3514$ & & - & 3596,3572 \\
\hline$v_{\text {as }}\left(\mathrm{NH}_{2}\right)$ & 3368 & 3410 & 3403 & 3421 & - \\
\hline$v_{s}\left(\mathrm{NH}_{2}\right)$ & 3163 & 3170 & 3158 & 3260 & - \\
\hline$v(\mathrm{CH})$ & 3060 & $3075,2984,2903$ & 3067 & - & - \\
\hline$v(C=O)$ & 1697,1679 & 1725 & 1708,1665 & 1660 & - \\
\hline$\delta(\mathrm{OH})$ & - & 1672 & - & - & - \\
\hline$\delta\left(\mathrm{NH}_{2}\right)$ & 1618 & 1620 & 1622 & 1625 & - \\
\hline$v(C=C)+v(C=N)$ & 1592,1573 & 1604,1569 & 1604,1578 & 1586 & - \\
\hline$v(\mathrm{C}-\mathrm{C})+\delta(\mathrm{C}-\mathrm{H})$ & 1484 & 1498,1480 & 1481 & - & - \\
\hline$v($ halka) & 1423 & 1448,1408 & 1436 & - & - \\
\hline$v(\mathrm{C}-\mathrm{N})_{\text {amit }}$ & 1395 & 1383 & 1378 & 1399 & - \\
\hline$v($ halka) & 1339 & 1327 & 1331 & - & - \\
\hline$\delta(\mathrm{CH})$ & 1254 & 1252 & 1247 & - & - \\
\hline$v$ (ring) & 1230 & 1228 & 1230 & - & - \\
\hline$v(\mathrm{C}-\mathrm{C})$ & 1201 & 1190 & 1207 & - & - \\
\hline$\delta\left(\mathrm{NH}_{2}\right)$ & 1153 & 1133 & 1157 & 1119 & - \\
\hline$v(\mathrm{C}-\mathrm{C})$ & 1123 & - & 1128 & - & - \\
\hline$\delta(\mathrm{CH})$ & 1090 & - & 1084 & - & - \\
\hline$v(\mathrm{~S}-\mathrm{O})$ & - & 1068 & - & - & 1070 \\
\hline Halka soluklanması & 1028 & - & 1061 & - & - \\
\hline v(halka) & 995 & 997 & 989 & - & - \\
\hline$\gamma(\mathrm{CH})$ & 970,936 & 949 & 951,935 & - & - \\
\hline$\gamma(\mathrm{OH})$ & - & 899 & - & - & - \\
\hline$\gamma(\mathrm{CH})$ & 829,778 & 802,752 & 820,775 & - & - \\
\hline$\delta$ (halka) & 702 & 711 & 727 & - & - \\
\hline$\delta(\mathrm{S}-\mathrm{O})$ & - & 684 & - & - & 600 \\
\hline $\mathrm{w}\left(\mathrm{NH}_{2}\right)$ & 644 & 644 & 658 & - & - \\
\hline$\delta$ (halka) & 622 & - & 630 & 641 & - \\
\hline$\delta(\mathrm{O}=\mathrm{CN})$ & 602 & 572 & 604 & - & - \\
\hline$\gamma(\mathrm{C}-\mathrm{H})$ & 510 & 517,499 & 548 & - & - \\
\hline$\gamma$ halka) & 433 & 444 & 443 & 436 & - \\
\hline$\gamma($ halka) & 411 & 424 & 412 & - & - \\
\hline
\end{tabular}

\section{Elektronik Özellikler}

$\mathrm{Su}$ içinde alınan kompleksin elektronik spektrumunda, $\mathrm{Cu}(\mathrm{II})$ metalin $\mathrm{d}$-d geçişine karşı1lı gelen 780 nm'de merkezlenmiş bir absorbsiyon bandı ortaya çıkar (Şekil 6). Bu değer, geniş bir zarf olarak ${ }^{2} \mathrm{~B}_{1 \mathrm{~g}} \rightarrow{ }^{2} \mathrm{E}_{\mathrm{g}}$ $\left(d_{x z, y z} \leftrightarrow d_{x}^{2}-{ }^{2}\right) \quad$ ve $\quad{ }^{2} B_{1 g} \rightarrow{ }^{2} B_{2 g} \quad\left(d_{x y} \leftrightarrow d_{x}{ }^{2}-{ }^{2}\right)$ geçişlerine atfedildi. X-1şını kırınımından görüldüğü gibi, bu geniş absorbsiyon band1 hafifçe bozulmuş oktahedral geometrinin varlığını gösterir. 300 nm'nin altındaki absorbsiyon bantları, na ligantlarının ve $\mathrm{SO}_{4}{ }^{2-}$ iyonlarının ligantlar arası yük geçişinden (ILCT, International Ligand Charge Transition) kaynaklanmaktadır. $\mathrm{Bu}$ bantlar, $\pi \rightarrow \pi^{*}$ ve $\mathrm{n} \rightarrow \pi^{*}$ geçişleri nedeniyle yaklaşık 200 ve 264 nm'de iki maksimum sergiler [9]. Ayrıca 360 $\mathrm{nm}$ de ki bant metal-ligant veya ligant-metal geçişinden (MLCT, Metal Ligand Charge 
Transition veya LMCT, Ligand Metal Charge Transition) kaynaklanmaktadır.

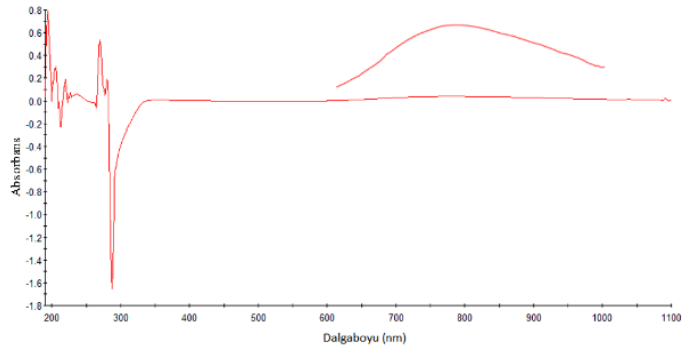

Şekil 6. Kompleksin UV-Vis spektrumu

\section{Termogravimetrik Analiz}

Kompleksin bozunması üç adımda gerçekleşmektedir (Şekil 7). İlk ağırlık kaybı, $115-225^{\circ} \mathrm{C}$ aralığında dört su molekülünün bozunması ile meydana gelir (gözlenen, \%10.01; hesaplanan, \%9.78). Nikotinamit literatürde $\quad 190-305^{\circ} \mathrm{C}$ sicaklık aralığında bozunurken [26], bu çalışmada kompleks, 225$375^{\circ} \mathrm{C}$ sıcaklık aralığında nikotinamit ve kalan su moleküllerini kaybeder. $\mathrm{Bu}$ aşamadaki deneysel kütle kayb1 \%44.61'dir (hesaplanan, \%44.02). Son aşamada, ekzotermik bir pik $\left(\mathrm{DTGmax}=736{ }^{\circ} \mathrm{C}\right) \mathrm{SO}_{4}{ }^{2-}$ iyonlarının yanmas1 nedeniyle olur. Son ürün olarak, $\left(\mathrm{CuO}_{2}\right)_{2}$ kalmıştır (gözlenen, \%25.66; hesaplanan, $\% 25.94)$.

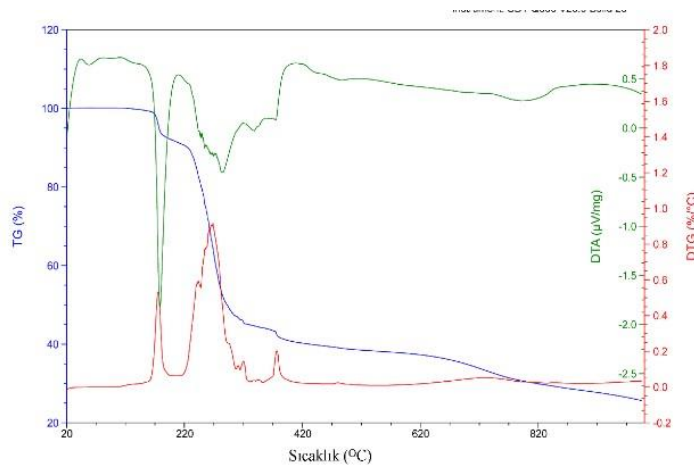

Şekil 7. Kompleksin TG (mavi çizgi), DTG (kırmızı çizgi) ve DTA (yeşil çizgi) ĕgrileri

\section{Sonuc}

$\mathrm{Bu}$ çalışmada yeni bir $\left[\mathrm{Cu}(\mathrm{na})_{2}\left(\mathrm{H}_{2} \mathrm{O}\right)_{4}\right]^{2+}$. $\left[\mathrm{Cu}\left(\mathrm{H}_{2} \mathrm{O}\right)_{6}\right]^{2+}$. $\left[\left(\mathrm{SO}_{4}\right)_{2}\right]^{4-} .\left(\mathrm{H}_{2} \mathrm{O}\right)_{2}$ kompleksi sentezlenmiş ve rapor edilmiştir. $\mathrm{Bu}$ kompleks $\mathrm{P} 2{ }_{1} / \mathrm{c}$ uzay grubunda kristalleşir. Yapısal çalışmasında nikotinamit ligantının amit azot atomları, $\left[\mathrm{SO}_{4}\right]^{2-}$ iyonu ve $\left[\mathrm{Cu}\left(\mathrm{HO}_{2}\right)\right]^{2+}$ katyonunu içeren hidrojen bağları ile üç boyutlu supramoleküler yap1 oluşumu vurgulamıştır. IR spektrumu serbest nikotinamit ligandı ile karşılaştırıldığında, hidrojen bağı ile bağlanan fonksiyonel grupların titreşim frekanslarında yukarı veya aşağı kaymalar görülmüştür. Kompleksin UVGörünür spektrumları geçiş metali etrafindaki koordinasyon ortamının bozulmuş oktahedral geometri olduğunu desteklemiştir. Termal analiz çalışmaları, nihai bozunma ürününün $\left(\mathrm{CuO}_{2}\right)_{2}$ olduğunu göstermektedir.

\section{Ek Materyal}

$\mathrm{Bu}$ çalışma için CCDC No: 1566697 ek kristalografik verileri içerir. $\mathrm{Bu}$ veri http://www.ccdc.cam.ac.uk/ adresinden ücretsiz olarak elde edilebilir.

Teşekkür -

Fon/Finansman bilgileri Yazar bu çalışmanın araştırması veya yayınlanması için herhangi bir mali destek almamıştır.

Etik Kurul Onayt ve İzinler -

Çıkar çatışmaları/Çatışan çıkarlar -

Yazarların Katkısı Yazar makalenin son halini okumuş ve onaylamıştır. 


\section{Kaynaklar}

[1] Ramalingam, S., Periandy, S., Govindarajan, M., \& Mohan, S. (2010). FT-IR and FT-Raman vibrational spectra and molecular structure investigation of nicotinamide: A combined experimental and theoretical study. Spectrochimica Acta Part A: Molecular and Biomolecular Spectroscopy, 75(5), 15528. https://doi.org/10.1016/j.saa.2010.02.015

[2] Kozlevčar, B., Leban, I., Turel, I., Šegedin, P., Petric, M., Pohleven, F., White, A. J. P., Williams, D. J., \& Sieler, J. (1999). Complexes of copper (II) acetate with nicotinamide: preparation, characterization and fungicidal activity; crystal structures of $\left[\mathrm{Cu}_{2}\left(\mathrm{O}_{2} \mathrm{CCH}_{3}\right)_{4}(\right.$ nia) $\left.)\right]$ and $\left[\mathrm{Cu}_{2}\left(\mathrm{O}_{2} \mathrm{CCH}_{3}\right)_{4}(\mathrm{nia})_{2}\right]$. Polyhedron, 18(5), 755-762. https://doi.org/10.1016/S0277-5387(98)00350-7

[3] Pirc, E. T., Modec, B., Cer-Kerčmar, K., \& Bukovec, P. (2014). Synthesis, structure, antioxidant and SOD-mimetic activity of $\left[\mathrm{Cu}\right.$ (xanthurenate)(nicotinamide) $\left.\left(\mathrm{H}_{2} \mathrm{O}\right)\right]$ complexes. Monatshefte für ChemieChemical Monthly, 145(6), 911-920. https://doi.org/10.1007/s00706-014-1168-y

[4] Castellano, E. E., Piro, O. E., Parajón-Costa, B. S., \& Baran, E. J. (2002). Crystal structure and vibrational behaviour of tetraaqua-di(nicotinamide) $\mathrm{M}(\mathrm{II})$-saccharinates, with $\mathrm{M}(\mathrm{II})=\mathrm{Co}, \mathrm{Ni}, \mathrm{Zn}$. Zeitschrift für Naturforschung B, 57(6), 657-660. https://doi.org/10.1515/znb-2002-0611

[5] Parajón-Costaa, B. S., Baran, E. J., Piro, O. E., \& Castellano, E. E. (2002). Crystal structure and vibrational behaviour of aqua di (saccharinato) di (nicotinamide) copper (II). Zeitschrift für Naturforschung B, 57(1), 43-46. https://doi.org/10.1515/znb-2002-0105

[6] Naïli, H., Rekik, W., Bataille, T., \& Mhiri, T. (2006). Crystal structure, phase transition and thermal behaviour of dabcodiium hexaaquacopper(II) bis(sulfate), $\left(\mathrm{C}_{6} \mathrm{H}_{14} \mathrm{~N}_{2}\right)\left[\mathrm{Cu}\left(\mathrm{H}_{2} \mathrm{O}\right)_{6}\right]\left(\mathrm{SO}_{4}\right)_{2}$. Polyhedron, 25(18), 3543-3554. https://doi.org/10.1016/j.poly.2006.07.010

[7] Saïd, S., Mhadhbi, N., Hajlaoui, F., Yahyaoui, S., Norquist, A. J., Mhiri, T., Bataille, T., \& Naïli, H. (2013). The structural transformation of monoclinic [(R)- $\left.\mathrm{C}_{5} \mathrm{H}_{14} \mathrm{~N}_{2}\right]\left[\mathrm{Cu}\left(\mathrm{SO}_{4}\right)_{2}\left(\mathrm{H}_{2} \mathrm{O}\right)_{4}\right] \cdot 2 \mathrm{H}_{2} \mathrm{O}$ into orthorhombic [(R)- $\left.\mathrm{C}_{5} \mathrm{H}_{14} \mathrm{~N}_{2}\right]_{2}\left[\mathrm{Cu}\left(\mathrm{H}_{2} \mathrm{O}\right)_{6}\right]\left(\mathrm{SO}_{4}\right)_{3}$ : crystal structures and thermal behavior. Phase Transitions, 87(1), 71-84. https://doi.org/10.1080/01411594.2013.787617

[8] Ma, J. F., Yang, J., Zheng, G. L., Li, L., \& Liu, J. F. (2003). A porous supramolecular architecture from a copper(II) coordination polymer with a 3d four-connected 86 net. Inorganic Chemistry, 42(23), 7531-7534. https://doi.org/10.1021/ic034846q

[9] Aycan, T. (2020). Hekzaakua-bis(sulfat) içeren nikotinamitli kobalt(II) ve çinko(II) koordinasyon bileşikleri: sentezlenmesi, yapısal, spektroskopik ve termal özelliklerinin incelenmesi. Geleceğin Dünyasında Bilimsel ve Mesleki Çalışmalar 2020/ Fen Bilimleri I, Ekin Basım Yayın Dağıtım, Bursa, 94-111.

[10] Sheldrick, G. (1992). SHELXS-97 and SHELXL-97, University of Gottingen, Germany.

[11] Farrugia, L. J. (1999). WinGX suite for small-molecule single-crystal crystallography. Journal of Applied Crystallography, 32(4), 837-838. https://doi.org/10.1107/S0021889899006020

[12] Farrugia, L. J. (1997). ORTEP-3 for windows-a version of ORTEP-III with a graphical user interface (GUI). Journal of Applied Crystallography, 30(5), 565-565. https://doi.org/10.1107/S0021889897003117

[13] Macrae, C. F., Edgington, P. R., McCabe, P., Pidcock, E., Shields, G. P., Taylor, R., Towler, M., \& Streek, J. v. d. (2006). Mercury: visualization and analysis of crystal structures. Journal of Applied Crystallography, 39(3), 453-457. https://doi.org/10.1107/S002188980600731X 
[14] Özbek, F. E., Sertçelik, M., Yüksek, M., Necefoğlu, H., Çelik, R. Ç., Nayir, G. Y., \& Hökelek, T. (2017). $\mathrm{Cu}(\mathrm{II})$ and $\mathrm{Ni}(\mathrm{II})$ 4-cyanobenzoate complexes with nicotinamide: Synthesis, spectral, structural and optical characterization and thermal behavior. Journal of Molecular Structure, 1150, 112-117. https://doi.org/10.1016/j.molstruc.2017.08.074

[15] Kaştaş, G., Aycan, T. A., Paşaoğlu, H., Kocabıyık, C., \& Karabulut, B. (2012). Supramolecular structures constructed by connection of one-dimensional polymer chains through the dimeric water clusters in coordination polymers of pyromellitate ligand. Journal of Inorganic and Organometallic Polymers and Materials, 22(5), 1003-1015. https://doi.org/10.1007/s10904-012-9686-8

[16] Etter, M. C. (1990). Encoding and decoding hydrogen-bond patterns of organic-compounds. Accounts of Chemical Research, 23(4), 120-126. https://doi.org/10.1021/ar00172a005

[17] Bernstein, J., Davis, R. E., Shimoni, L., \& Chang, N. L. (1995). Patterns in hydrogen bonding functionality and graph set analysis in crystals. Angewandte Chemie International Edition, 34(15), 1555-1573. https://doi.org/10.1002/anie.199515551

[18] Paşaoğlu, H., Güven, S., Heren, Z., \& Büyükgüngör, O. (2006). Synthesis, spectroscopic and structural investigation of $\mathrm{ZnI}_{2}$ (nicotinamide) $)_{2}, \mathrm{ZnI}_{2}$ (isonicotinamide) ${ }_{2}$ and $\left.\left[\mathrm{Zn}\left(\mathrm{H}_{2} \mathrm{O}\right)_{2} \text { (picolinamide }\right)_{2}\right] \mathrm{I}_{2}$. Journal of Molecular Structure, 794(1), 270-276. https://doi.org/10.1016/j.molstruc.2006.02.045

[19] Bayarı, S., Ataç, A., \& Yurdakul, Ş. (2003). Coordination behaviour of nicotinamide: an infrared spectroscopic study. Journal of Molecular Structure, 655(1), 163-170. https://doi.org/10.1016/S00222860(03)00256-4

[20] Stuart, B. H. (2004). Infrared Spectroscopy: Fundamentals and Applications, Wiley.

[21] Ak Aycan T., Paşaoğlu, H., \& Kaştaş, G. (2016). Two different coordination dimers of pyromellitic acid in terms of molecular geometry and supramolecular architecture. Journal of Molecular Structure $1105(1), 238-245$.

[22] Sundaraganesan, N., Ilakiamani, S., \& Joshua, B. D. (2007). FT-Raman and FT-IR spectra, ab initio and density functional studies of 2-amino-4, 5-difluorobenzoic acid. Spectrochimica Acta Part A: Molecular and Biomolecular Spectroscopy, 67(2), 287-297. https://doi.org/10.1016/j.saa.2006.07.016

[23] Tyagi, S., Singh, S. M., Gencaslan, S., Sheldrick, W., \& Singh, U. P. (2002). Metal-5-fluorouracilhistamine complexes: solution, structural, and antitumour studies. Metal-based drugs, 8(6), 337-345. https://doi.org/10.1155/MBD.2002.337

[24] Secco, E. A. (1988). Spectroscopic properties of $\mathrm{SO}_{4}$ (and $\mathrm{OH}$ ) in different molecular and crystalline environments. I. Infrared spectra of $\mathrm{Cu}_{4}(\mathrm{OH})_{6} \mathrm{SO}_{4}, \mathrm{Cu}_{4}(\mathrm{OH})_{4} \mathrm{OSO}_{4}$, and $\mathrm{Cu}_{3}(\mathrm{OH})_{4} \mathrm{SO}_{4}$. Canadian Journal of Chemistry, 66(2), 329-336. https://doi.org/10.1139/v88-057

[25] Nakamoto, K. (2009). Infrared and Raman Spectra of Inorganic and Coordination Compounds, Part B: Applications in Coordination, Organometallic, and Bioinorganic Chemistry. Wiley.

[26] Köse, D., \& Necefoğlu, H. (2008). Synthesis and characterization of bis (nicotinamide) mhydroxybenzoate complexes of $\mathrm{Co}(\mathrm{II}), \mathrm{Ni}(\mathrm{II}), \mathrm{Cu}(\mathrm{II})$ and $\mathrm{Zn}(\mathrm{II})$. Journal of Thermal Analysis and Calorimetry, 93(2), 509-514. 